\title{
Asp 280 residue is important in the activity of the Escherichia coli leader peptidase
}

\author{
Meesook Sung ${ }^{1,3}$ and Kwangsook Park ${ }^{2}$ \\ 1 Biochemistry Department, College of Medicine, Kwandong University, Kangnung \\ 210-701, Korea \\ 2 Department of Microbiology and Institute for Viral Diseases, College of Medicine, \\ Korea University, Seoul 136-705, Korea \\ 3 Corresponding author
}

Accepted 27 March 1999

Abbreviations: SDS, sodium dodecyl sulfate; IPTG, isopropyl-1-thio- $\beta$-Dgalactopyranoside; OmpA, outer membrane protein A; Lep, leader peptidase

\section{Abstract}

Leader peptidase is a novel serine protease in Escherichia coli, which catalyzes the cleavage of amino-terminal signal sequences from exported proteins. It is an integral membrane protein containing two transmembrane segments with its carboxyterminal catalytic domain residing in the periplasmic space. Recently, the $x$-ray crystal structure of signal peptidase-inhibitor complex showed that Asp 280, a highly conserved consensus sequence of $E$. coli leader peptidase is the closest charged residue in the vicinity of two catalytic dyad, Ser 90 and Lys 145 , and it is likely held in place by a salt bridge to Arg 282. Possible roles of Asp 280 and Arg 282 in the structure-catalytic function relationship were investigated by the site-directed mutagenesis of Asp 280 substituted with alanine, glutamic acid, glycine, or asparagine and of Arg $\mathbf{2 8 2}$ with methionine. All of mutants purified with nickel affinity chromatography were inactive using in vitro assay. It is surprising to find complete lose of activity by an extension of one carbon units in the mutant where Asp 280 is substi-tuted with glutamic acid. These results suggest that Asp 280 and Arg 282 are in a sequence which constitutes catalytic crevice of leader peptidase and are essential for maintaining the conformation of catalytic pocket.
\end{abstract}

Keywords: Escherichia coli, leader peptidase (signal peptidase) site-directed mutagenesis

\section{Introduction}

Leader(signal) peptidase is a member of novel class of serine proteinases which utilize serine and lysine residues for catalysis (Sung and Dalbey, 1992; Tschantz et al., 1993), and an essential protein in both prokaryotic and eukaryotic cells. It has been purified from Escherichia coli (Zwizinski et al., 1980), yeast (YaDeau and Blobel, 1989), chicken (Baker and Lively, 1987), and dog (Greenburg et al., 1989), and identified in Salmonella typhimurium (van Dijl et al., 1990), Pseudomonas fluorescens (Black et al., 1992) and Bacillus subtilis (van Dijl et al., 1992). Gene cloning and mutagenesis techniques have been employed to study the membrane biogenesis of leader peptidase and to determine its physiological role (Dalbey and Wickner, 1985 and 1987). The active site of the leader peptidase residues in the periplasmic domain, which is anchored to the membrane by two transmembrane segments (Figure 1A; Bilgin et al., 1990). It consists of a single polypeptide chain with a molecular weight of $35,988 \mathrm{Da}$ (323 amino acid residues), contains one disulfide bond, and has an isoelectric point of 6.9 (Wolfe et al., 1983).

Recently, there has been a very important development in understanding the catalytic mechanism of leader peptidase. Paetzel et al. (1998) have solved the x-ray cystal structure of an Escherichia coli leader peptidase ( $\Delta 2-75, \mathrm{Mr} 27,952 \mathrm{Da}, 249$ amino acids) which retains catalytic activity. The structure has been determined at $1.9 \AA$ resolution with a $5 \mathrm{~S}, 6 \mathrm{~S} \beta$-lactam inhibitor covalently bound as an acyl enzyme intermediate to Ser 90 . In the structure of leader peptidase, aspartic acid 280 is the closest charged group in the vicinity of catalytic active sites, serine 90 and Lys 145, and it is likely held in place by a salt bridge to arginine 282 (Figure 1). In addition, there are four highly conserved regions of sequence which have been classified within the catalytic domain of signal peptidases commonly termed Box B, C, D, and E (Dalbey et al., 1997). Box $E$ contains the highly conserved residues, Asp 280 and Arg 282 (Table 1).

The study of catalytic mechanism of leader peptidase has been specially emphasized in the antibacterial drug design since the location of the bacterial leader peptidase in either the periplasm or extracellular space makes accessible target for antibiotics. In order to identify a possible role of Asp 280 in the E. coli leader peptidase protein, we generated mutant proteins with specific substitutions using site-directed mutagenesis. The result of our study suggests that Asp 280 residue plays an important role in stabilizing $E$. coli leader peptidase structure, possibly, through salt bridges to Arg 282, however, it has not excluded the possibility of the catalytic role of Asp 280 in the leader peptidase protein. 

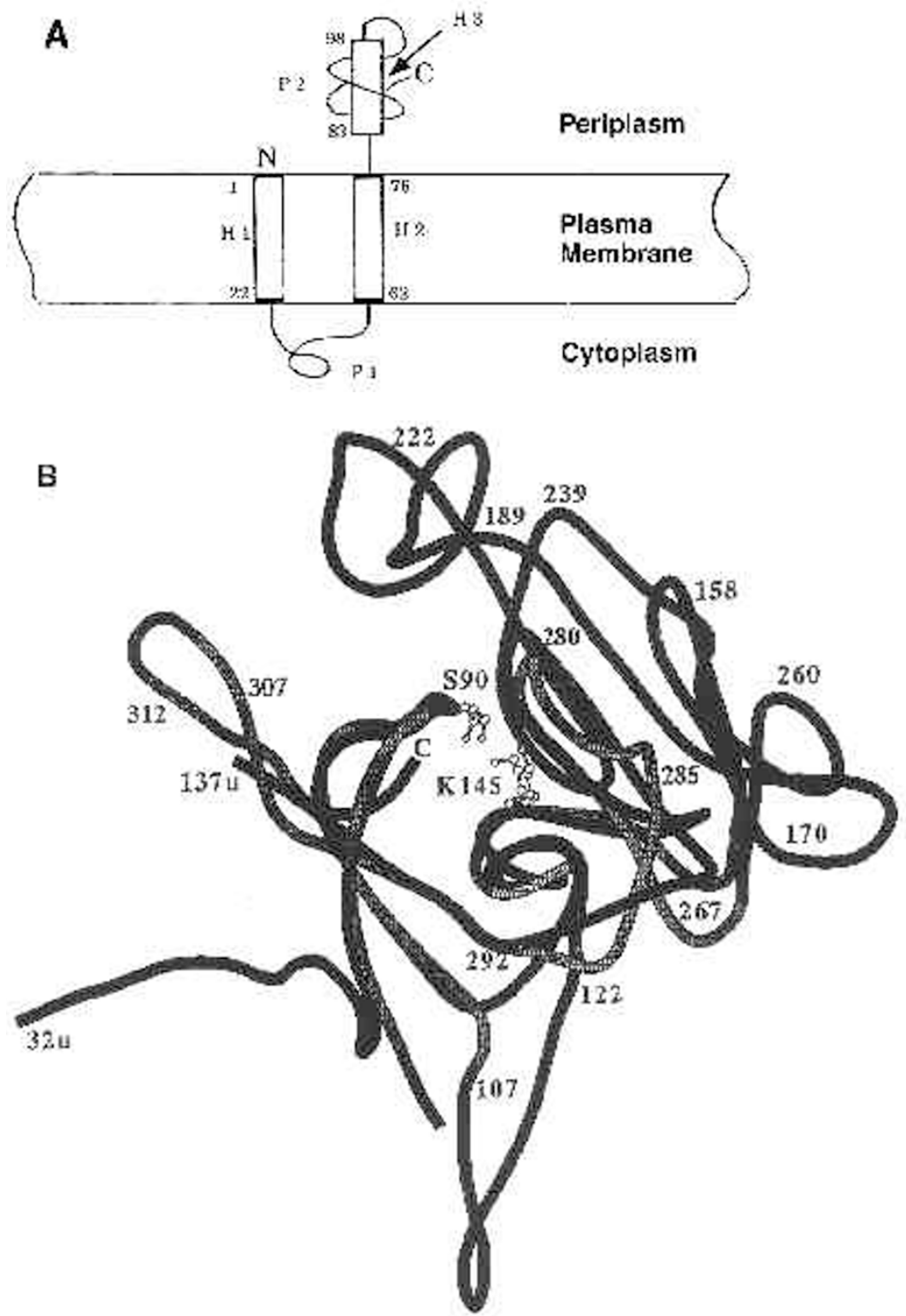

Figure 1. A. Membrane topology of $E$. coli leader peptidase. $\mathrm{H} 1, \mathrm{H} 2$, and $\mathrm{H} 3$ are hydrophobic domains, and $\mathrm{P} 1$ and $\mathrm{P} 2$ are polar domains. B. A ribbon diagram depicting the general fold of leader paptidase $\Delta r$ 2-75. The Asp 280 is the nearest residue around the catalytic active sites, Ser 90 and Lys 145 . The figure is reproduced from Paetzel et al., 1998.

\section{Materials and Methods}

\section{Materials}

Oligonucleotides were synthesized at the IDT(Integrated DNA Technologies, Inc., IA, U.S.A.). The Ni-NTA resin was purchased from Qiagen Inc. (CA, U.S.A.).

\section{Bacterial strains and plasmids}

E. coli strains, SB221 and MC1061 were from the Ohio State University. The cloning of the pro-OmpA nuclease A gene into the IPTG-inducible plasmid pONF1 (Takahara et al., 1985) and the overexpression of the protein were described by Chatterjee et al. (1995). Oligonucleotidedirected mutagenesis was used to engineer 6 consecutive histidine residues into the cytoplasmic domain of leader peptidase that can purify the mutants from the chromosome-expressed wild-type leader peptidase. Amino acid residues at the position 35-40 were substituted with histidine residues. The sequence of the oligonucleotide used is as follows: 5'-TTC GCA CCT AAA CGG CGG CGC GAA CGT CAT CAT CAT CAT CAT CAT GCT CGG GAC TCA CTG GAT AAA GCA- 3'.

\section{Purification of the 6-His Tagged Leader Peptidase Proteins}


The 6-His tag/nickel affinity chromatography was used to purify the overexpressed mutant leader peptidases away from the wild-type chromosome-expressed copies of leader peptidase. All leader peptidase proteins including the wild-type, contained the 6 -His residues. E. coli MC1061 cells containing the pET plasmid encoding the mutant leader peptidase protein were grown in LB media (2L) containing $100 \mu \mathrm{g} / \mathrm{ml}$ ampicillin and $0.2 \%$ sucrose until an absorbance of 0.45 at $600 \mathrm{~nm}$ was reached. Expression was induced by the addition of arabinose to a final concentration of $0.2 \%$, and the incubation of the cultures was continued for $4 \mathrm{~h}$. The cells were pelleted and then resuspended in an equal weight of $50 \mathrm{mM}$ Tris, $\mathrm{pH} 7.5,10 \%$ sucrose. The cells were frozen by dropping them into liquid nitrogen and stored at $-80^{\circ} \mathrm{C}$ until required. Marble-sized nuggets of frozen cell suspension $(30 \mathrm{~g})$ were thawed by addition to $100 \mathrm{ml}$ of buffer (10 mM Tris-CI, pH 8.5, 5 mM EDTA, 20\% sucrose) rapidly stirring at $23^{\circ} \mathrm{C}$. Lysozyme $(20 \mathrm{mg})$ and DNase I (2 mg) were added and the suspension was stirred for $30 \mathrm{~min}$ at room temperature. It was then frozen in a bath of dry ice and ethanol, thawed in a bath of tap water, mixed with $1 \mathrm{ml}$ of $1 \mathrm{M}$ magnesium acetate, and stirred for $30 \mathrm{~min}$ at room temperature. The solution was then centrifuged at $18,000 \mathrm{rpm}\left(4^{\circ} \mathrm{C}\right.$, $30 \mathrm{~min}$ ), and the pellet was resuspended in $30 \mathrm{ml}$ buffer (10\% glycerol, $10 \mathrm{mM}$ triethanolamine chloride, $\mathrm{pH} 7.5$ ) and centrifuged again. The membrane pellet was resuspended at $0^{\circ} \mathrm{C}$ in binding buffer $(5 \mathrm{mM}$ imidazole, $0.5 \mathrm{M} \mathrm{NaCl}, 20 \mathrm{mM}$ Tris- $\mathrm{HCl} \mathrm{pH}$ 8.0, $1 \%$ Triton X-100, $10 \mathrm{mM} \beta$-mercaptoethanol) with a Dounce homogenizer. Detergent-insoluble material was removed by centrifugation at $18,000 \mathrm{rpm}$. The Triton X-100 extract was applied at $4^{\circ} \mathrm{C}$ to a $1 \mathrm{ml}$ nickel column which was equilibrated with the binding buffer. The column was then washed with $20 \mathrm{ml}$ of binding buffer followed by a second wash with wash buffer $(60 \mathrm{mM}$ imidazole, $0.5 \mathrm{M}$ $\mathrm{NaCl}, 10 \mathrm{mM}$ Tris, $\mathrm{pH} 8.0,1 \%$ Triton X-100, $10 \mathrm{mM} \beta$ mercapto-ethanol). The 6-His tagged leader peptidase was eluted by an imidazole gradient buffer $(100,200$, 300,400 , and $500 \mathrm{mM}$ imidazole each, $0.5 \mathrm{M} \mathrm{NaCl}, 20$ $\mathrm{mM}$ Tris- $\mathrm{HCl}, \mathrm{pH}$ 8.0). The eluted fraction was collected in $1 \mathrm{ml}$ each concentration, assayed for protein by SDS-PAGE and visualized by Coomassie brilliant blue staining. Fractions containing leader peptidase activity were pooled and stored at $-80^{\circ} \mathrm{C}$.

\section{Purification of the pro-OmpA nuclease $A$}

The E. coli strain SB221 bearing the plasmid pONF1 was used to overexpress the pro-OmpA nuclease $A$ substrate, which is a hybrid protein of staphylococcal nuclease $A$ fused to the signal peptide of the outer membrane protein (OmpA) (Takahara et al., 1985). The cells were grown to the late log phase in M9-casamino acids medium supple-mented with $5 \mu \mathrm{g} / \mathrm{ml}$ leucine and 5 $\mu \mathrm{g} / \mathrm{ml}$ tryptophan. Pro-OmpA nuclease A was induced for $4 \mathrm{~h}$ by addition of $2 \mathrm{mM}$ IPTG and purified as described by Chatterjee et al. (1995) and used as substrate for estimating the activity of leader peptidase.

\section{Assay for the Processing of Pro-OmpA Nuclease A}

Reaction mixture $(13.5 \mu \mathrm{l})$ containing $1 \mu \mathrm{l}$ of leader peptidase (final concentration $0.364 \mu \mathrm{M}$ ) was incubated at $37^{\circ} \mathrm{C}$ with $9 \mu \mathrm{L}$ of pro-OmpA nuclease $\mathrm{A}(6 \mu \mathrm{M})$ in 50 $\mathrm{mM}$ Tris, $\mathrm{pH} 8.0$ in the presence of $1 \%$ Triton $\mathrm{X}-100$. The reaction was terminated by adding $5 \times$ dye supplemented with $100 \mathrm{mM} \mathrm{MgCl}_{2}$, 5\% SDS, 25\% glycerol, $200 \mathrm{mM} \beta$ mercaptoethanol, $200 \mathrm{mM}$ Tris ( $\mathrm{pH} \mathrm{6.8)} \mathrm{and} \mathrm{immediately}$ freezing the mixture at $-70^{\circ} \mathrm{C}$. The samples were analyzed on $17 \%$ SDS-polyacrylamide and the gel was stained with Coomassie Brilliant Blue. The concentration of ProOmpA nuclease was determined at $280 \mathrm{~nm}$ ( $E$ at $280 \mathrm{~nm}$ $=8.31$ ).

\section{Western blot analysis}

$0.5 \mu \mathrm{g}$ of purified leader peptidase proteins was electrophoresed in a $17 \%$ SDS polyacrylamide gel and the proteins in the gel were then electroblotted to a nitrocellulose membrane. The membrane was treated with the antirabbit polyclonal leader peptidase antibody $(1: 10,000$ dilution) and then with alkaline phosphatase (Pierce, IL, U.S.A.). The membrane was visualized using nitro blue tetazolium chrolide and 5-bromo-4-chloro-indoyl phosphate according to the manufacturer's protocol.

\section{Results}

Since it is likely that Asp 280 residue is critical to signal peptidase structure and function, each of mutants was constructed and purified to evaluate for a possible role of D280 using an in vitro assay. We have designated XNY ( $\mathrm{X}$, amino acids in the wild-type protein; $\mathrm{N}$, the position of the amino acids; $Y$, the new substituted amino acids). The sequence of the mutagenic primers are shown in Table 2, and the sites of mutations can be seen in the working model of leader peptidase in Figure 1B. The genes encoding these mutant proteins were subcloned into the pET-23d vector, which is a T7 polymerase-dependent used in the expression of high levels of protein. The mutant proteins were then purified using a nickel affinity column as described in 'Materials and Methods'. The yield of each purified mutant was approximately the same. Typically, $4.6 \mathrm{mg}$ of purified mutants was obtained from $1 \mathrm{~L}$ cell culture. Each of purified proteins, WT (lane 1), D280A (lane 2), D280E (lane 3), D280G (lane 4), D280N (lane 5), and R282M (lane 6) were shown in Figure 2A. The wild-type of leader peptidase was purified to its homogeneity but all of the mutant proteins were shown additional protein bands. In order to see antigenic 
$A$

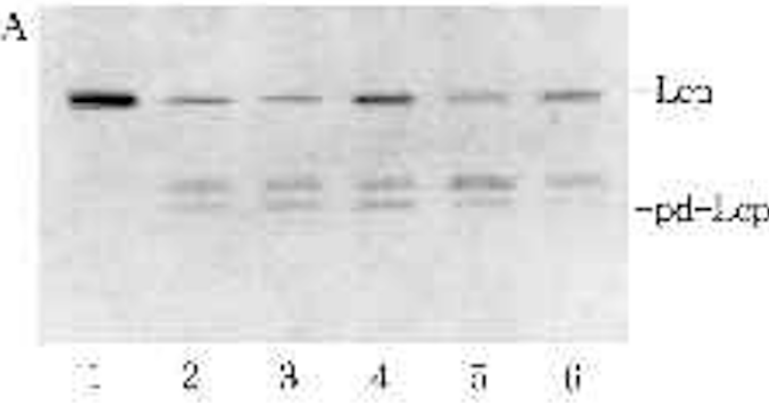

Q

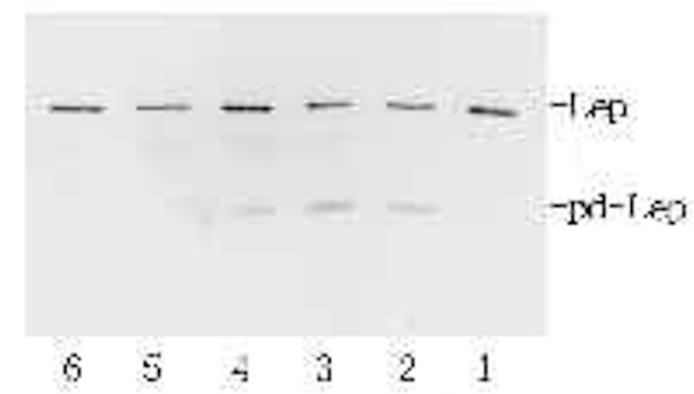

Figure 2. A. Purification of the mutant leader peptidases. WT (lane 1), D280A (lane 2), D280E (Lane 3), D280G (lane 4), D280N (lane 5), and R282M (lane 6), each of $0.5 \mu \mathrm{g}$, leader peptidase were applied to a SDS-PAGE $(17 \%)$, and the gel was stained by Coomassie Brilliant Blue. B. Western blot analysis of the mutant leader peptidases. The proteins in the gel as indicated in panel $A$ were reacted with the anti-rabbit polyclonal leader peptidase antibody and the color reaction was developed as described in the 'Materials and Methods'. Lep indicates the position of wild-type and mutant leader peptidases and pd-Lep indicates the position of partiallly degraded mutant leader peptidases.

reactivity of these purified mutant proteins, the western blot analysis was performed using anti-rabbit polyclonal leader peptidase antibody. The additional band (bottom band in Figure $2 \mathrm{~B}$ ) as well as the top band showing the whole structure of protein is also detected in the Asp 280 mutants, suggesting that it is the partially degraded $E$. coli leader peptidase protein. Since no additional band was seen in the wild-type and G272A mutant leader peptidase (data not shown), we believe that the utation of D280 impacts on the stable structure of leader peptidase protein and, therefore, the unstable protein is easily degraded during purification procedures.

Activity of mutant leader peptidases was assayed (Figure 3) using pro-OmpA nuclease A, an excellent substrate for the wild-type leader peptidase (Chatterjee et al., 1995). In comparison of the activity of wild-type leader peptidase with background activity, this assay system is 10 fold more sensitive than that of in vitro system that previously was used to show the serine 90 and lysine 145 are possible active site residues (Sung and Dalbey, 1992; Tschantz et al., 1993). An aliquot containing 0.364 $\mu \mathrm{M}$ (undiluted) of each purified leader peptidase proteins,
Table 1. Conserved domain (E) of leader(signal) peptidases.

\begin{tabular}{|c|c|c|}
\hline & & * \\
\hline Lep (Eco) & 272 & GDNRDNSADSR \\
\hline Lep (Sty) & 273 & GDNRDNSADSR \\
\hline Lep (Pfl) & 225 & GDNRDNSNDSR \\
\hline Lep (Hin) & 297 & GDHRDHSDDSR \\
\hline Lep (Rca) & 207 & GDNRDNSEDSR \\
\hline Sip (Bja) & 195 & GDNRDNSADSR \\
\hline Lep (Pla) & 160 & GDNRNNSYDSH \\
\hline Sip (Mtu) & 237 & GDNRTHSADSR \\
\hline SpsB(Sau) & 146 & GDNREVSKDSR \\
\hline Sips (Bsu) & 145 & GDNRRNSMDSR \\
\hline SipT (Bsu) & 154 & GDNRLNSMDSR \\
\hline SipU (Bsu) & 148 & GDNRLNSLDSR \\
\hline SipP (pTA1015) & 147 & GDNRQESMDSR \\
\hline SipP (pTA1040) & 146 & GDNRQNSMDSR \\
\hline Sips (Bam) & 146 & GDNRRNSMDSR \\
\hline SipT (Bam) & 154 & GDNRLNSMDSR \\
\hline Sip (Bli) & 147 & GDNRQRSMDSR \\
\hline Sip (Bca) & 144 & GDNRLSSWDSR \\
\hline Imp1p & 130 & GDNLSHSLDSR \\
\hline Imp2p & 123 & GDNYFHSIDSN \\
\hline Sip (Mja) & 138 & GDNNPI HDPE \\
\hline Sec11 & 102 & GDNNAG-NDIS \\
\hline Spc18 & 115 & GDN--NAVDDR \\
\hline Spc21 & 127 & GDN--NEVDDR \\
\hline
\end{tabular}

Bam, Bacillus amyloliquefaciens; Bca, Bacillus caldolyticus; Bja, Bradyrhizobium japonicum; Bli, Bacillus licheniformis; Bsu, Bacillus subtilis; Eco, Escherichia coli; Hin, Haemophilus influenzae; Imp, Inner membrane protease; Lep, leader peptidase; Mja, Methanococcus jannasxhii; Mtu, Mycobacterium tuberculosis; Pfl, Pseudomonas fluorescens; Pla, Phormidium laminosum; Rca, Rhodobacter capsulatus; Sau, Staphylococcus aureus; Sip, signal peptidase; SPC, signal peptidase complex; Sty, Salmonella typhimurium.

after dilution $\left(1: 10,1: 10^{2}, 1: 10^{3}, 1: 10^{4}, 1: 10^{5}\right)$ with a buffer (20 mM Tris- $\mathrm{HCl}, \mathrm{pH} 8.0)$ was incubated with the pro-OmpA nuclease $A$ substrate at $37^{\circ} \mathrm{C}$ for $30 \mathrm{~min}$ and analyzed by SDS-PAGE and Coomassie Brilliant Blue staining. All of the mutant leader peptidases except D280G had $1 / 10^{3}$ the activity of wild-type protein corresponding to background enzymatic activity. It is interesting to note that the D280G mutant leader peptidase had a lower processing activity $\left(1 / 10^{2}\right.$ to $1 / 10^{3}$ fold activity of wild-type protein). Since the residues, aspartic acid 280 and arginine 282 are possibly connected by a salt bridge, we believe that D280 and $\mathrm{R} 282$ residues are important in stabilizing the whole structure of leader peptidase protein. Three double leader peptidase mutants were made by remaining the charges (D280E, R282K), exchanging two residues (D280R, R282D), and engineering disulfide bond (D280C, 


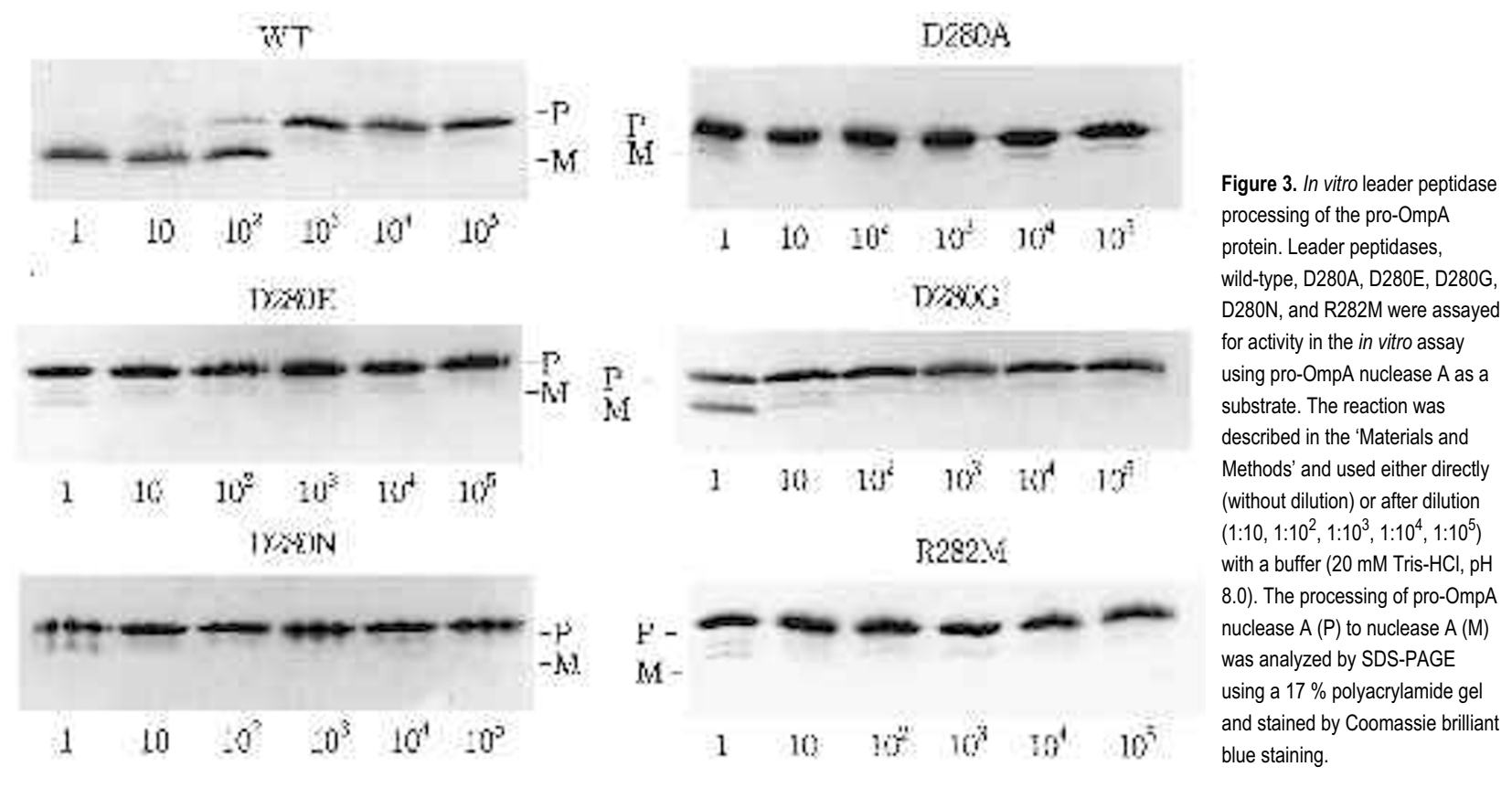

Table 2. Sequence of leader peptidase mutants of the Lep B gene.

\begin{tabular}{ll}
\hline Sequence of oligonucleotides & Changes \\
\hline GACAACAGCGCGGCCAGCCGTTACTGGGGC & D280A \\
GACAACAGCGCGGAGAGCCGTTACTGGGGC & D280E \\
GACAACAGCGCGGGCAGCCGTTACTGGGGC & D280G \\
GACAACAGCGCGAACAGCCGTTACTGGGGC & D280N \\
GACAACAGCGCGGACAGCCGTTACTGGGGC & D280M \\
GACAACAGCGCGGTGAGCTGTTACTGGGGC & D280C, R282C \\
GACAACAGCGCGGAGAGCAAGTACTGGGGC & D280E, R280K \\
GACAACAGCGCGCGCAGCGATTACTGGGGC & D280R, R282D \\
\hline
\end{tabular}

The codons, which were changed to make the mutations, are underlined.

$\mathrm{R} 282 \mathrm{C}$ ) to mimic the bridge. All of three mutant leader peptidases were purified and assayed using pro-OmpA substrate. They also had a very low activity as the other single mutants (data not shown).

\section{Discussion}

Bacterial leader peptidases are not inhibited by any of the standard inhibitors to serine, cysteine, metallo-, or aspartic proteases (Zwizinski et al., 1981). Furthermore, extensive site-directed mutagenesis and chemical modification studies have revealed that bacterial leader peptidases have an essential Ser and Lys residue, but no essential His or Cys residues (Black MT, 1993; van Dijl et al., 1995). Based on these studies, it has been proposed that leader peptidases belong to a unique class of serine proteases which utilize a mechanism whereby the lysine acts as the general base to extract the proton from the hydroxyl side-chain of the serine.
Since Asp 280 and Arg 282 residues of E. coli leader peptidase are possibly connected by salt bridges around the catalytic active sites, Ser 90 and Lys 145, and they are the highly conserved residues in the Box $\mathrm{E}$ domain, it has been proposed that the residues possibly make essential contributions to the stability of active site or function of the protein. In this study, the single (D280A, D280E, D280G, D280N, and R282M) or double [(D280C, R282C), (D280E, R282K) and (D280R, R282D)] mutant leader peptidases are purified with a nickel affinity column and assayed using an in vitro assay, resulting in an inactive enzyme. This shows that the Asp 280 and Arg 282 residues in $E$. coli leader peptidase play a critical role in the activity of the protein. All of D280 mutant leader peptidases show additional two bands on Coomassie brilliant blue staining gel but one additional band on the western blot analysis. Since wild-type of purified protein shows homogeneous one band without an additional 
band, we think that the D280 mutant produces additional bands by impacting the stable whole structure of protein. The purified R282M mutant also shows an additional band in the Coomassie brilliant blue staining gel like D280 mutants but not in the western blot analysis, suggesting that the additional band could be the product of impure leader peptidase protein rather than the partially degraded protein. When we performed CD spectroscopy on the purified K145A, the result of CD spectrum is similar to that of the wild-type protein (Tschantz et al., 1993). From the fact that there is no large conformational change even in K145A mutant and the $\mathrm{x}$-ray structural aspect of Asp 280 with a salt bridge to R282, it is unlikely that Asp 280 mutant proteins are inactive as the result of global conformational changes. In order to show that there were no conformational changes within the mutant leader peptidases, a competition study between inactive D280 mutants and wild-type of leader peptidase for substrate binding will be taken as one of additional studies. It is also unlikely that Asp 280 play a role as a catalytic triad observed in the classic serine proteinases since it would require a significant substrate induced conformational change to make the Lys145 - Asp 280 interaction (Paetzel et al., 1998). In this stage, we speculate that Asp 280 provides primarily a structural role in maintaining the integrity of the active site residues.

In summary, there are unanswered questions regarding the exact mechanism of the leader peptidase. It is clear from in-vitro study that Asp 280 and Arg 282 are critical residues for the activity of leader peptidase protein, and it does not yet definitely provide the evidence as a proposed structure role. The forthcoming $x$-ray crystal structure of the soluble fragment of $E$. coli leader peptidase may contribute to answer this question regarding the detail catalytic mechanism of leader peptidase.

\section{Acknowledgement}

We thank Dr. Ross E. Dalbey and his laboratory in the Ohio State University for his expert advice and supplying materials.

\section{References}

Baker, R. K. and Lively, M. O. (1987) Purification and characterization of chicken oviduct microsomal signal peptidase. Biochemistry 26: 8561-8567

Bilgin, N., Lee, J. I., Zhu, H.-Y., Dalbey, R. E. and von Heijne, G. (1990) Mapping of catalytically important domains in Escherichia coli leader peptidase. EMBO J. 9: 27172722
Black, M. T., Munn, J. G. R., Allsop, A. E. (1992) On the catalytic mechanism of prokaryotic leader peptidase. Biochem. J. 282: 539-543

Black, M. T. (1993) Evidence that the catalytic activity of prokaryote leader peptidase depends upon the operation of a serine-lysine catalytic dyad. J. Bacteriol. 172: 4957-4961

Chatterjee, S., Suciu, D., Dalbey, R. E., Kahn, P. C. and Inouye, M. (1995) Determination of $\mathrm{Km}$ and kcat for signal peptidase I using a full length secretory precursor, pro-OmpA-nuclease A. J. Mol. Biol. 245: 311-314

Dalbey, R. E. and Wickner, W. (1985) Leader peptidase catalyzes the release of exported proteins from the outer surface of the Escherichia coli plasma membrane. J. Biol. Chem. 260: 15925-15931

Dalbey, R.E. and Wickner, W. (1987) Leader peptidase of Escherichia coli: critical role of a small domain in membrane assembly. Science 235: 783-787

Dalbey, R. E., Lively, M. O., Bron, S. and van Dijl, J. M. (1997) The chemistry and enzymology of the type I signal peptidases. Protein Science 6: 1129-1138

Greenburg, G., Shelness, G. S. and Blobel, G. (1989) A subunit of mam-malian signal peptidase is homologous to yeast SEC11 protein. J. Biol. Chem. 264: 15762-15765

Paetzel, M., Dalbey, R. E. and Strynadka, N. C. J. (1998) Crystal structure of E. coli type 1 signal peptidase-inhibitor complex at 1.9 Å resolution, submitted

Sung, M. and Dalbey, R. E. (1992) Identification of potential active site residues in the Escherichia coli leader peptidase. J. Biol. Chem. 267: 13154-13159

Takahara, M., Hibler, D., Barr, P. J., Gerlt, J. A. and Inouye, M. (1985) The OmpA signal peptide directed secretion of staphylococcal nuclease A by E. coli. J. Biol. Chem. 260: 2670-2674

Tschantz, W. R., Sung, M., Delgado-Partin, V. M. and Dalbey, R. E. (1993) A serine and a lysine residue implicated in the catalytic mechanism of the Escherichia coli leader peptidase. J. Biol. Chem. 268: 27349-27354

van Dijl, J. M., van den Bergh, R., Reversma, T., Smith, H., Bron, S. and Venema, G. (1990) Molecular cloning of the Salmonella typhimurium lep gene in Escherichia coli. Mol. Gen. Genet. 223: 233-240

van Dijl, J. M., de Jong, A., Vehmaanper J, Venema, G. and Bron, S. (1992) Signa peptidase I of Bacillus subtilis: Patterns of conserved amino acids in prokaryotic and eukaryotic type I signal peptidases. EMBO J. 11: 2819-2828

van Dijl, J. M., de Jong, A., Venema, G. and Bron, S. (1995) Identification of the potential active site of the signal peptidase SipS of Bacillus subtilis. J. Biol. Chem. 270 3611-3618

Wolfe, P. B., Wickner, W. and Goodman, J. M. (1983) Sequence of the leader peptidase gene of Escherichia coli and the orientation of leader peptidase in the bacterial envelop. J. Biol. Chem. 258: 12073-12080

YaDeau JT and Blobel G. (1989) Solubilization and characterization of yeast signal peptidase. J. Biol. Chem. 264: 2928-2934

Zwizinski, C. and Wickner W. (1980) Purification and characterization of leader (signal) peptidase from Escherichia coli. J. Biol. Chem. 255: 7973-7977

Zwizinski, C., Date, T. and Wickner W. (1981) leader peptidase is found in both the inner and outer membranes of Escherichia coli. J. Biol. Chem. 256: 3593-3597 


\section{Retraction}

Vol. 31 (1999) 64-69

\section{Asp 280 residue is important in the activity of the Escherichia coli leader peptidase}

Meesook Sung ${ }^{1,3}$ and Kwangsook Park ${ }^{2}$

${ }^{1}$ Biochemistry Department, College of Medicine, Kwandong University, Kangnung 210-701, Korea

${ }^{2}$ Department of Microbiology and Institute for Viral Disease, College of Medicine, Korea University, Seoul 136-705, Korea

${ }^{3}$ Corresponding author: Tel, 82-391-649-7464; Fax, 82-391-641-1074; E-mail, msung @kdccs.kwandong.ac.kr

The above article was retracted by the authors because of irreproducible in vitro assay data. 